\title{
STRATIGRAPHIE, NANNOFOSSILES CALCAIRES ET FORAMINIFÈRES DE LA COUPE DU RUISSEAU DE LESPONTES À SAINT-LON-LES-MINES (EOCÈNE MOYEN ET SUPÉRIEUR D'AQUITAINE, FRANCE)
}

\author{
STRATIGRAPHY, CALCAREOUS NANNOFOSSILS AND FORAMINIFERA \\ OF THE LESPONTES RIVER SECTION \\ AT SAINT-LON-LES-MINES (MIDDLE AND UPPER EOCENE \\ OF AQUITAINE, FRANCE)
}

\author{
par Etienne STEURBAUT*, Kàroly SZTRÀKOS** et Dirk NOLF***
}

\begin{abstract}
RÉsumÉ. - L'étude détaillée de divers affleurements le long du Ruisseau de Lespontes et ses affluents à Saint-Lon-les-Mines permet de reconnaître trois unités essentiellement marneuses dans l'Eocène moyen et supérieur du Synclinal du Bassecq. L'unité inférieure est constituée par des marnes bathyales, attribuables à la Formation des Marnes de Miretrain et d'âge lutétien supérieur - bartonien inférieur (NP 15b, NP 15c, NP 16 et base NP 17 ; EPA 9 à EPA 11 (? EPA 12) et milieu de la P 11 - sommet de la P 12). Elle est surmontée par un complexe de marnes essentiellement gréseuses et glauconieuses, de milieu circalittoral, inconnu jusqu'à présent. Ces marnes fortement tectonisées sont appelées ici Marnes de Bagats et sont définies comme nouveau membre de la Formation des Marnes de la Côte des Basques. Elles chevauchent la limite bartonienne/priabonienne sensu Berggren et al., 1995 (sommet NP 17 et base NP 18 ; EPA 13 et partie médiane de P 14). L'unité supérieure comprend des marnes finement sableuses, dans lesquelles, vers le sommet de la coupe, viennent s'intercaler des bancs calcaires. Ces Marnes de Brihande appartiennent à la partie inférieure du Priabonien (NP 18, vraisemblablement jusqu'au sommet de la coupe ; EPA 13, EPA 14 et en partie EPA 15, sommet P 14 et en partie P 15). La série du Ruisseau de Lespontes est interprétée en termes de stratigraphie séquentielle, permettant de préciser ses rélations à l'échelle du Bassin de l'Adour. L 'intégration des données démontre qu'en Aquitaine l'apparition de Chiasmolithus oamaruensis (base de la zone NP 18) est nettement antérieure à l'apparition de Globigerinatheka semiinvoluta (base de la zone P 15) et que l'identification de l'intervalle à nannofossiles NP 19 - NP 20 y est difficile, vu la rareté de l'espèce marqueur Isthmolithus recurvus.
\end{abstract}

ABSTRACT. - Detailed analysis of a series of outcrop sections along the Lespontes River and its tributaries at Saint-Lon-les-Mines has led to the identification of three predominantly marly units within the Middle and Upper Eocene of the Bassecq Syncline. The lowermost unit consists of bathyal marls, attributable to the Miretrain Marl Formation, and is of Late Lutetian - Early Bartonian age (NP 15b, NP 15c, NP 16 and base NP 17 ; EPA 9 to EPA 11 (? EPA12) and middle P 11 to top P 12). It is overlain by circalittoral, glauconitic indurate marls unknown up to now. This marly, intensely fractured unit, called the Bagats Marl Member, is defined to represent a new member within the Côte des Basques Marl Formation. It encompasses the Bartonian/Priabonian boundary sensu Berggren et al., 1995 (top NP 17 and base NP 18 ; EPA 13 and middle P 14). The uppermost unit is made up of fine sandy marls, containing several limestone intercalations towards the top. These Brihande Marls are included in the lower part of the Priabonian (NP 18, probably up to the top of the section ; EPA 13, EPA 14 and part of EPA 15, top P 14 and in part P 15). The succession of the Lespontes River is interpreted in terms of sequence stratigraphy, allowing refining its relationship within the Adour Basin. The integration of the data indicates that in Aquitaine the appearance of Chiasmolithus oamaruensis (base of zone NP 18) is prior to the appearance of Globigerinatheka semiinvoluta (base of zone P 15) and that nannofossil interval NP 19 - NP 20 is very difficult to identify in this area, due to the scarcity of the marker species Isthmolithus recurvus. France

Mots-clés : Eocène moyen et supérieur - Nannofossiles calcaires - Foraminifères - coupe du Ruisseau de Lespontes - Aquitaine - SW

Key-words : Middle and Upper Eocene - Calcareous nannofossils - Foraminifera - Lespontes River section - Aquitaine - SW France

* Institut royal des Sciences Naturelles de Belgique (IRScNB), 29 rue Vautier, B-1000 Bruxelles, Belgique \& KULeuven, Redingenstraat 16, B-3000 Leuven, Belgique; Etienne.Steurbaut@naturalsciences.be.

** 35 rue Savier, F-92240 Malakoff, France ; e-mail : karoly_sztrakos@hotmail.com.

*** Institut royal des Sciences Naturelles de Belgique, 29 rue Vautier, B-1000 Bruxelles, Belgique ; Dirk.Nolf@natuurwetenschappen.be. 


\section{INTRODUCTION}

Le Ruisseau de Lespontes, affluent de l'Adour, recoupe la série éocène à Saint-Lon-les-Mines sous forme de nombreux petits affleurements, parfois en ravinant de grandes falaises de plus de $30 \mathrm{~m}$ d'hauteur (Fig. 1). Cette coupe se situe dans le Synclinal du Bassecq (Kieken et Thibault, 1975), à mi-chemin entre Dax et Peyrehorade. Les affleurements s'étendent à l'ouest du centre de St-Lon-les-Mines, entre la ferme Luscly, $0.5 \mathrm{~km}$ au sud-est de Bagats (point 1 ; feuille 1343 est ; $\mathrm{x}=318.600, \mathrm{y}=3151.850)$ et le point où le ruisseau change de direction, $2 \mathrm{~km}$ plus à l'ouest (point A ; $x=316.800, y=3153.375$ ) (Fig. 2).

La coupe du Ruisseau de Lespontes a été étudiée par Boulanger (1968) et lors du levé de la carte géologique de Saint-Vincent-de-Tyrosse (Kieken et Thibault, 1975). Au début des années 80 Nolf a échantillonné le gisement de Bignaou sur la rive droite du Lespontes (point $\mathrm{P}$ sur la Fig. 2) dans le cadre de l'étude des otolithes éocènes de l'Aquitaine. Ces mêmes échantillons ont été analysés par Steurbaut en 1984 qui les a rattachés à la zone à nannofossiles calcaires NP 20, vu la présence de Sphenolithus pseudoradians, marqueur de la base de la NP 20 (données publiées dans Nolf, 1988). A partir de 1985 plusieurs auteurs (Perch-Nielsen, 1985 ; Martini et Müller, 1986) ont démontré que S. pseudoradians apparaît plus tôt dans l'échelle de temps, et par conséquent, qu'on ne peut plus distinguer la zone NP 20 telle quelle, mais seulement l'intervalle NP 19-20.

Des échantillons difficilement localisables, pris par feu D. Boulanger, ont été revus par Sztràkos (2000). Cette révision a montré la présence de deux formations dans le secteur : les Marnes de Miretrain d'âge lutétien et les Marnes de Brihande d'âge priabonien. La compilation des données issues des échantillons de Boulanger (DB) et des nouvelles récoltes de Nolf et Steurbaut essentiellement entre 1993 et 1996 montrent que la stratigraphie de cette coupe est plus complexe.

\section{DESCRIPTION DES COUPES}

Le début de la série étudiée, représentée par des marnes bleuâtres, est largement exposé dans le lit d'un affluent du Ruisseau de Lespontes, venant de Lartigue et passant au sud de la ferme Luscly (points 1 à 3 , fig. 2). La coupe se poursuit dans le talus de la route vers le centre de Saint-Lon-les-Mines, à environ cent $\mathrm{m}$ au nord du pont sur le Ruisseau de Lespontes (point 4, fig. 2 ) et le long du ruisseau, cent $\mathrm{m}$ plus au nord-ouest (point Z). A environ $30 \mathrm{~m}$ au sud du hangar de l'usine de Bagats, on observe une alternance de marnes hémipélagiques bleuâtres et de niveaux calcaires dans une petite falaise (coupe 0 ,

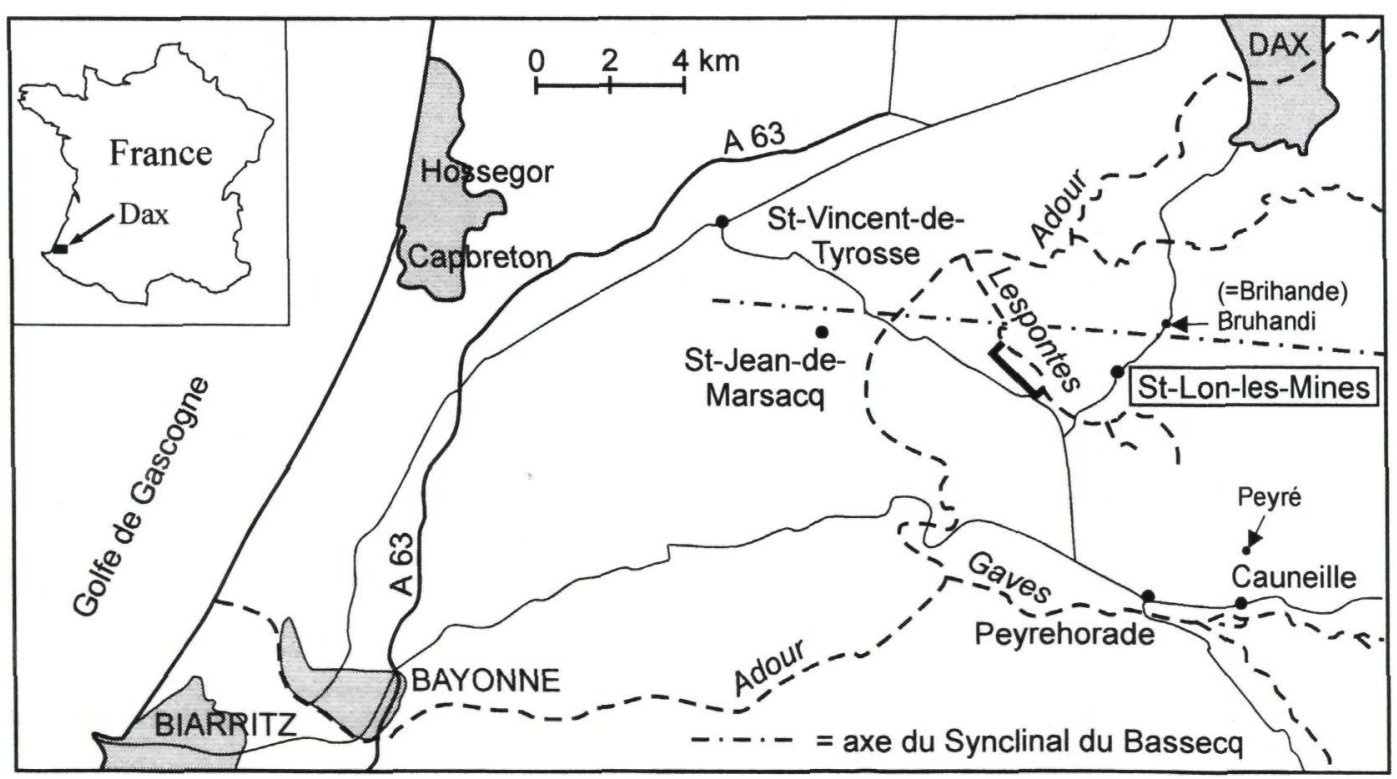

Fig. 1. Carte de l'Aquitaine méridionale entre Dax et Biarritz et localisation de la coupe du Ruisseau de Lespontes. Map of southern Aquitaine between Dax and Biarritz and location of the Lespontes River section. 


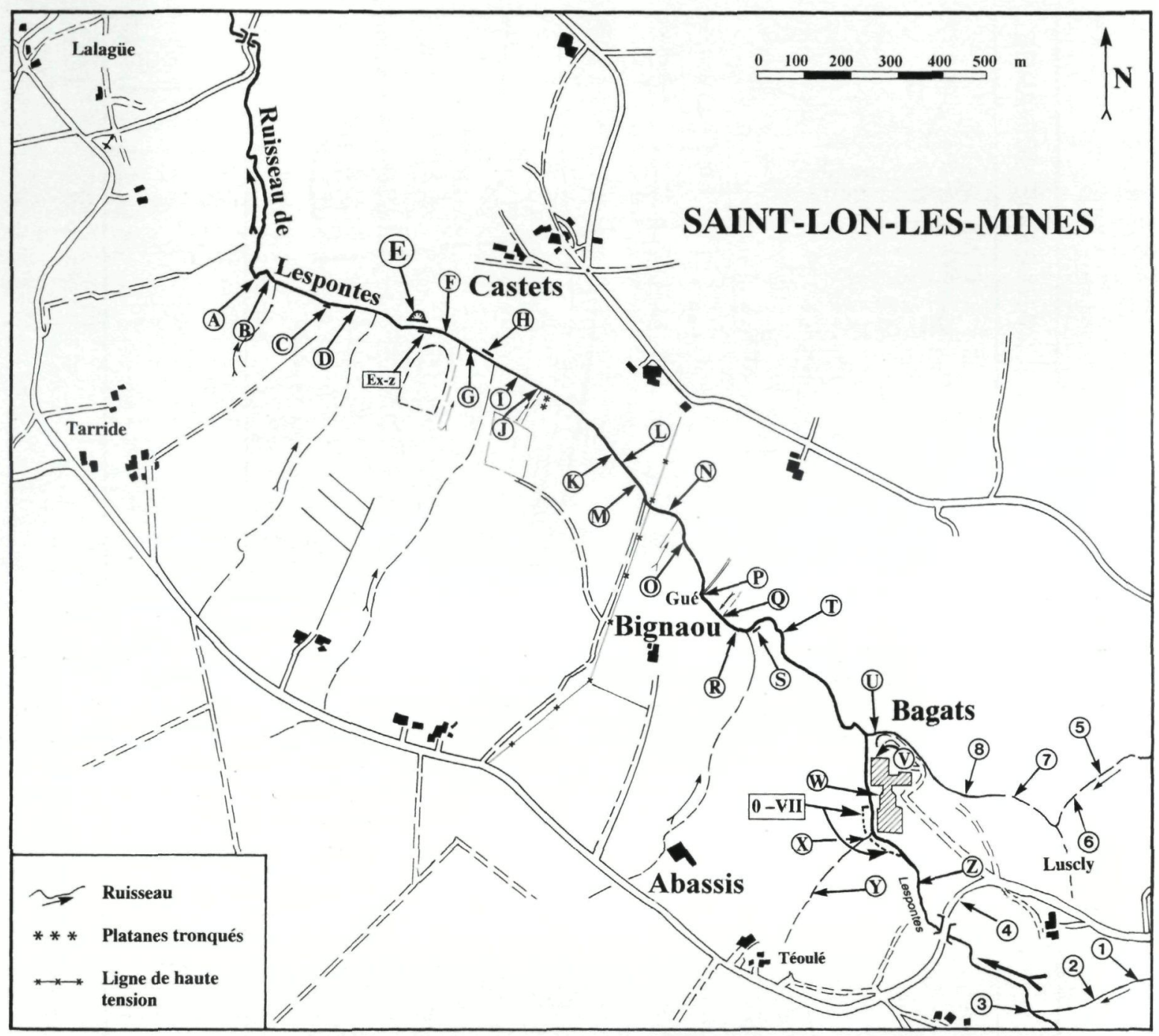

FIG. 2. Carte des affleurements sur le Ruisseau de Lespontes et ses affluents au NW du centre de Saint-Lon-les-Mines. Map of the exposures on the Lespontes River and its tributaries, NW of the Saint-Lon-les-Mines village center.

fig. 3). La série de marnes, qu'on a pu suivre à partir du pont jusqu'à cette falaise, est attribuable aux Marnes de Miretrain. Elle est surmontée par des dépôts essentiellement glauconieux, inconnus jusqu'à présent et appelés ici Marnes de Bagats. Leur composition et leur signification sont discutées ci-dessous. Les premiers niveaux glauconieux se rencontrent dans la coupe II, $10 \mathrm{~m}$ en amont de la cascade située au SW de l'usine de Bagats. On n'a pas pu observer le contact avec les Marnes de Miretrain sous-jacentes. Celui-ci se situe entre la coupe 0 et la coupe II. Le contact avec les Marnes de Brihande sus-jacentes a été identifié dans la coupe IV, $40 \mathrm{~m}$ en aval de la cascade. Il s'agit d'une surface bioturbée, séparant des marnes sableuses grisâtres, glauconieuses, grossières et fossilifères à leur base (otolithes de poissons, dents de requins, coquilles, quelques gros grains de glauconie) du banc gréseux glauconieux sous-jacent. Cette surface de non-dépôt, surmontée par un niveau condensé fossilifère (éch. IV a), représente la base des Marnes de Brihande. Les gisements IV et W constituent done les premiers affleurements des Marnes de Brihande, dont les niveaux stratigraphiques se succèdent au nord de l'usine de Bagats, en descendant le cours du Lespontes. Les débris de mollusques y sont nombreux (Cerithium sp., Turritella sp., bi valves), ainsi que des restes de bryozoaires, échinodermes et poissons. Les grands foraminifères déplacés 


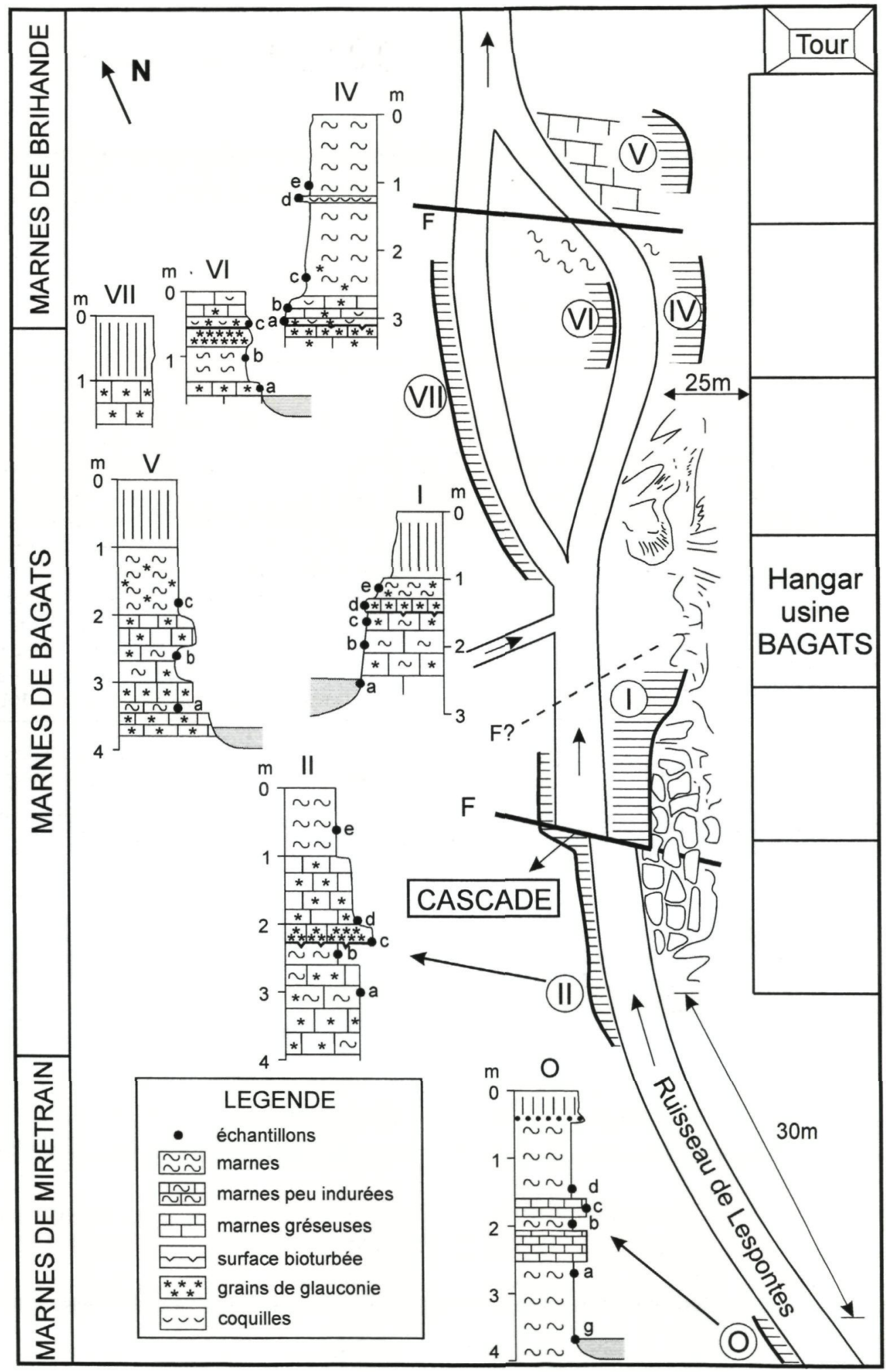

Fig. 3. Localisation et stratigraphie des coupes situées du côté ouest de l'usine de Bagats. Location and stratigraphy of the sections on the west of the Bagats factory. 
(nummulites, discocyclines) apparaissent localement. Ces mêmes couches (sommet des Marnes de Miretrain : points 5 et 6 , les Marnes de Bagats : point 7 , et la base des Marnes de Brihande : point 8) se retrouvent à l'est de l'usine de Bagats, dans le lit d'un petit affluent du Ruisseau de Lespontes, passant au N de Luscly. La falaise $\mathrm{E}$, située sur la rive droite du Ruisseau de Lespontes, à environ $1,4 \mathrm{~km}$ en aval de l'usine de Bagats, représente la partie la plus élevée de la coupe, avec l'alternance de marnes finement sableuses et de calcaires $(\mathrm{x}=317.125 ; \mathrm{y}=3153.250)$. Ces alternances marquent la transition vers les Calcaires de Siest (pour la coupe et la position des échantillons dans la falaise, voir Nolf et al., ce volume, fig. 8).

\section{MEMBRE DES MARNES DE BAGATS}

Localité-type : Saint-Lon-les-Mines, au sud-ouest du hangar de l'usine de Bagats (feuille 1343 est ; $\mathrm{x}=318.025 ; \mathrm{y}=3152.175$; fig. 2 et 3 ).

Définition : marnes gréseuses et grès, très glauconieux alternant avec des marnes bleuâtres, moins glauconieuses.

Limites : le secteur de l'usine de Bagats est fortement tectonisé, à cause de la proximité de l'accident de Saint-Lon-les-Mines. Par ce fait, la limite inférieure du nouveau membre, vers les Marnes de Miretrain, est inconnue. Sa limite supérieure est également incertaine à cause de son passage progressif dans les Marnes de Brihande. Ainsi, les échantillons c et e de la coupe IV, rattachés à ce member, contiennent encore de la glauconie et peuvent être sableux (Fig. 3). Nous avons retenu la surface bioturbée sous l'échantillon IV a, décrite dans le chapitre précédent, comme limite entre les deux membres.

Remarques : Les Marnes de Bagats, représentées par des faciès détritiques et glauconieux, appartiennent à une nouvelle unité au sein de la Formation des Marnes de la Côte des Basques. Elles sont les equivalents latéraux des Marnes de la Villa Marbella supérieures plus argileuses, dépourvues de la fraction sableuse et de glauconie. L'apparition des grès dans le Synclinal de Bassecq est liée à la phase tectonique compressive que 1'on situe entre les unites Palassou II et Pallassou III. Elle provoque la remontée et l'érosion simultanée des diapirs et des lames triasiques qui entourent ce synclinal : Dax, Tercis-lesBains au nord et Biarotte au sud-ouest. Les cristaux de quartz bipiramidés spécifiques aux dépôts de cet âge ont été observés dans le Membre de Bagats.
Les Marnes de Bagats appartiennent à un prisme de bas niveau, comparable au Complexe chaotique d'Ilbarritz et de l'Horizon à lumachelles de la Côte des Basques (Gély et Sztràkos, 2000, p. 48). L'abondance de la glauconie à gros grains et des restes de poissons, ainsi que la présence des surfaces durcies dans les coupes I, II et IV sont les indices d'une sédimentation condensée. L'absence des figures sédimentaires caractéristiques des faciès côtiers (stratification oblique, etc.) et la présence des foraminifères circalittoraux-épibathyaux, comme Martinottiella, Karreriella, Oridorsalis, Faboplanulina ammophila, etc., indiquent un milieu de dépôt distal sous une tranche d'eau dont l'épaisseur dépasse les $100 \mathrm{~m}$.

L'alternance des niveaux gréseux et marneux semble être provoquée par la capture périodique des sediments grossiers glauconieux en amont, en relation avec les mouvements tectoniques et du diapirisme dans la proximité de l'anticlinal de Saint-Lonles-Mines. Des niveaux détritiques, glauconieux sont connus dans d'autres parties du Bassin de l'Adour. Ainsi, on peut observer une couche à de nombreux remaniements (quartz triasique, silex crétacés, foraminifères d'âge divers) et à encroûtements glauconieux dans la marnière de Miretrain, au sommet de la formation du même nom à Angoumé où elle se place dans la zone NP 17, P 14 et EPA 12 (Sztràkos et al., 1998). A Cagnotte, à la ferme Tobacco (éch. DB 505), un horizon glauconieux s'observe dans les mêmes biozones (Sztràkos, 2000).

Age : Les affleurements de la localité-type appartiennent aux zones NP 17 supérieure - NP 18 inférieure en terme de nannoplancton et aux zones $\mathbf{P} 14$ et EPA 13 dans l'échelle des foraminifères planctoniques.

\section{LES NANNOFOSSILES CALCAIRES}

La taxinomie adoptée est en principe celle de Perch-Nielsen (1985), tenant compte des modifications apportées par Varol (1992) (Sullivania) et Aubry (1999) (Blackites). Tous les échantillons de la coupe du Ruisseau de Lespontes contiennent de riches associations de nannofossiles calcaires, aussi bien de point de vue qualitatif que quantitatif. Elles sont marquées par la coexistence de formes épicontinentales et de formes plus océaniques, permettant de reconnaître la biozonation de Martini (1971 : zones NP) ainsi que celle de Okada et Bukry (1981 : zones CP), établie en région océanique. L'interprétation biostratigraphique présentée ici est essentiellement basée sur les premières apparitions, et non sur les 
disparitions, vu le grand nombre de formes remaniées. A cela s'ajoute que le matériel étudié montre toujours une certaine dissolution, parfois suffisamment forte pour gêner les déterminations spécifiques. De plus, certains marqueurs sont extrêmement rares dans les associations d'Aquitaine, ce qui rend leur datation encore plus difficile. Isthmolithus recurvus, marqueur de la base de la zone NP 19 et espèce fréquente dans le Bassin de la Mer du Nord (Vandenberghe et al., 2003) n'a été retrouvé que dans deux échantillons sur dix d'âge priabonien supérieur (Biochrone NP 19-20) dans les coupes de Biarritz (Mathelin, 1988, fig. 36-38 ; Mathelin et Sztràkos, 1993, p. 29) et dans un seul niveau (St-Jean-de-Marsacq, marnière de Peyrelongue) sur huit d'âge éocène supérieur étudié par Lézaud (1967). Nous n'avons rencontré cette espèce en grand nombre que dans le gisement de Peyré à Cauneille (feuille 1443 ouest ; $\mathrm{x}=325.525, \mathrm{y}=3146.125)$, en association avec Chiasmolithus oamaruensis, Discoaster saipanensis, Pontosphaera aff. zigzag, Sphenolithus tribulosus, Pemma spp. et de rares Cribrocentrum reticulatum et Clausicoccus subdistichus. Il en ressort que l'intervalle NP 19-20 est difficile à reconnaître en Aquitaine. L'ensemble de nos données, bien entendu assez incomplet, suggère que les apparitions de $P$. aff. zigzag et de Clausicoccus subdistichus semblent très proches de celle de $I$. recurvus et peuvent donc être utilisées pour marquer la base de l'intervalle NP 19-20 en Aquitaine.

La partie basale de la coupe (échantillons 1 à 3 ) est caractérisée par la présence de Blackites gladius, Sullivania gigas, Lophodolithus acutus, Discoaster wemmelensis, Campylosphaera dela et Sphenolithus furcatolithoides, permettant une attribution à la zone NP 15b et la zone CP 13b. Parmi ces espèces, S. gigas et $D$. wemmelensis ne sont plus représentées dans l'échantillon sus-jacent 4 . Celui-ci en diffère en

\section{PLANCHE 1}

Tous les spécimens figurés proviennent de la coupe du Ruisseau de Lespontes à Saint-Lon-les-Mines. Les lames et négatives des photos de la planche 1 sont conservées dans les archives de l'Institut Royal des Sciences naturelles de Belgique, rue Vautier 29, B-1000 Bruxelles, Belgique. Les numéros donnés aux spécimens figurés y refèrent. La barre sur chaque photo représente 5 um. Abréviations utilisées : MEB = microscope électronique à balayage ; n.p. = nicols parallèles, fond clair ; n.c. $=$ nicols croisés, fond noir .

1. Blackites gladius (LOCKER, 1967) Varol, 1989 ; MEB ; éch. Z ; NP 15c (IRScNB b4079).

2. Blackites gladius (LOCKER, 1967) Varol, 1989 ; MEB ; éch. Z ; NP 15e (IRScNB b4080).

3. Reticulofenestra umbilica (LEVIv, 1965) Martini et Ritzkowski, 1968 ; MEB ; éch. Z ; NP 15e (IRSeNB b4081).

4. Pontosphaera sp. ; MEB ; éch. V ; NP 18 (IRScNB b4082).

5. Helicosphaera papillata BUKRY et BRAMLETTE, 1969 ; MEB ; éch. Z ; NP15e (IRScNB b4083).

6. Discoaster saipanensis BRAMLETTE et RIEDEL, 1954 ; MEB ; éch. Z ; NP15c (IRScNB b4084).

7. Coccolithus formosus (KAMPTNER, 1963) Wise, 1973 ; MEB ; éch. G ; NP 18 (IRScNB b4085).

8. Chiasmolithus sp. ; MEB ; éch. V b ; NP 18 (IRScNB b4086).

9. Pontosphaera prava (LOCKER, 1967) Romein, 1979 ; MEB ; éch. Od ; NP 16 (IRScNB b4087).

10. Helicosphaera salebrosa PERCH-Nielsen, 1971 ; MEB ; éch. Vb ; NP 18 (IRSeNB b4088).

11. Cribrocentrum reticulatum (GARTNer et SMith, 1967) PerchNielsen, 1971 ; n.c. ; éch. Ia ; NP 18 (IRScNB b4089).

12. Pedinocyclus larvalis (BUKRY et BRAMLETTE, 1969) Loeblich et Tappan, 1973 ; a = n.p. ; b=n.c. ; éch. IIe ; NP 17 (IRSeNB b4090).

13. Lanternithus minutus STRADNER, 1962 ; MEB ; éch. G ; NP 18 (IRSeNB b4091).

14. Sphenolithus moriformis (BRöNnimanN et STRADNER, 1960) Bramlette et Wilcoxon, 1967 ; MEB ; éch. G ; NP 18 (IRScNB b4092).
15. Corannulus germanicus STRADNER, 1962 ; MEB : éch. G : NP 18 (IRScNB b4093).

16. Neococcolithes minutus (PERCH-NIELSEN, 1967) Perch-Nielsen, 1971 ; n.c. ; éch. IVd ; NP 18 (IRScNB b4094).

17. Chiasmolithus oamaruensis (DEFLANDRE, 1954) Hay, Mohler et Wade, 1966 ; n.p. ; éch. Ia ; NP 18 (IRScNB b4095).

18. Chiasmolithus oamaruensis (DEFLANDRE, 1954) Hay, Mohler et Wade, $1966 ; \mathbf{a}=\mathbf{n} . p$. ; b = n.c. ; éch. IVe ; NP 18 (IRScNB 4096).

19. Lithostromation deflandrei LEZAUD, 1968 ; n.p. ; a = plan focal bas ; $b=$ plan focal haut ; éch. IVd ; NP 18 (IRSeNB b4097).

20. Helicosphaera wilcoxonii GARTNER, 1971 ; n.c. ; éch. IId ; NP 17 (IRScNB b4098).

21. Helicosphaera compacta BraMLette et Wilcoxon, 1967 ; n.c. ; éch. Id ; NP 18 (IRSeNB b4099).

22. Sullivania sp. ; MEB ; éch. Od ; NP 16 (IRScNB b4100).

23. Clathrolithus spinosus MARTINI, 1961 ; n.p. ; a = plan focal bas ; b = plan focal haut ; éch. Ia ; NP 18 (IRSeNB b4101).

24. Dictyococcites bisectus (HAY, MoHLER et WADE, 1966) Bukry et Percival, 1971 ; n.c. ; éch. Ia ; NP 18 (IRScNB b4102).

25. Chiasmolithus grandis (BRAMLETTE et RIEDEL, 1954) Radomski, 1968 ; n.c. ; éch. IId ; NP 17 (IRSeNB b4103).

26. Chiasmolithus grandis (BRAMLETTE et RIEDEL, 1954) Radomski, 1968 ; n.c. ; éch. I d ; NP 18 (IRSeNB b4104).

27. Sphenolithus spiniger BUKRY, 1971 ; MEB ; éch. Od ; NP 16 (IRSeNB b4105).

28. Zygrhablithus bijugatus (DEFLANDRE in DeFlandre et Fert, 1954) Deflandre, 1959 ; MEB ; éch. G ; NP 18 (IRScNB b4106). 

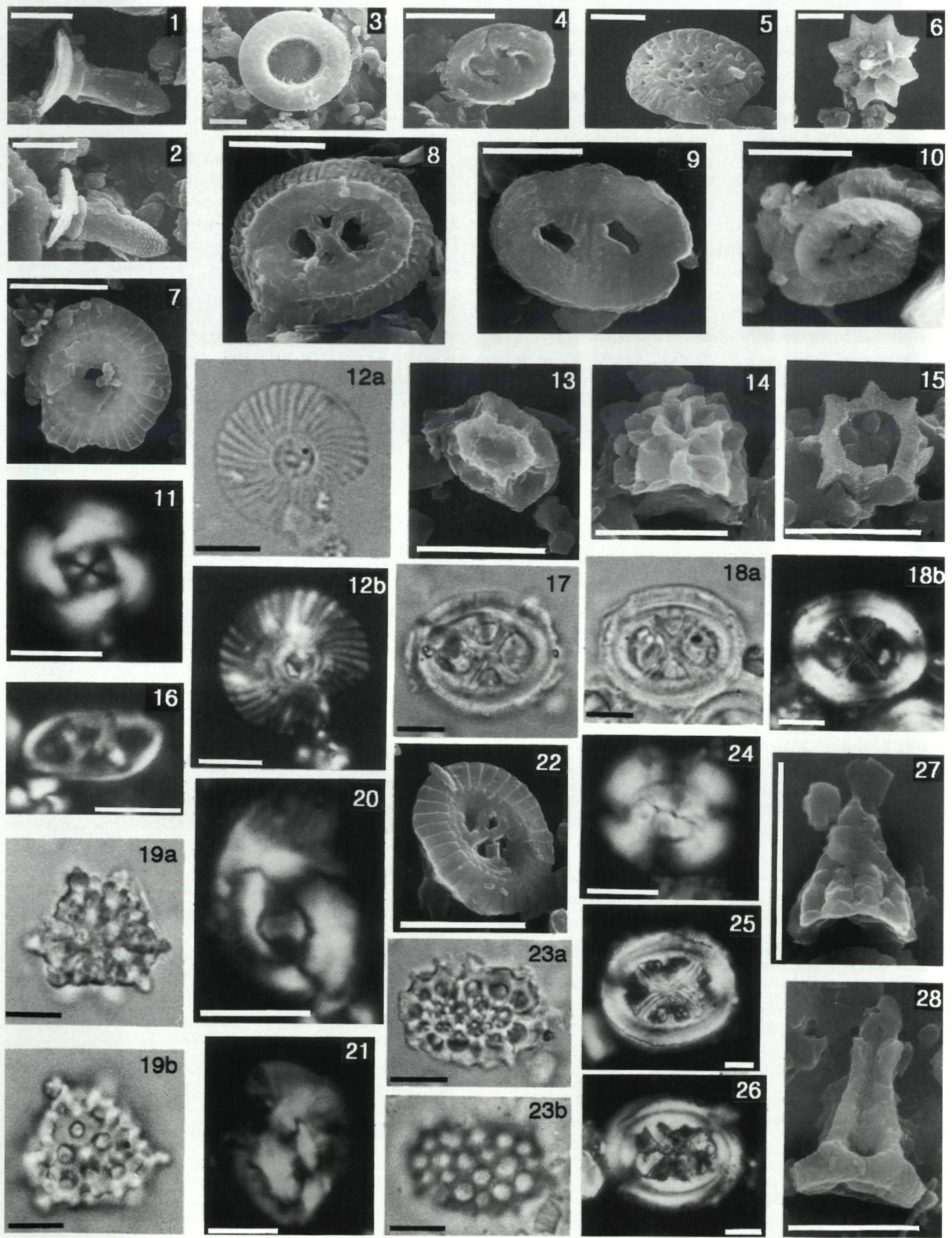

REVUE DE MICROPALÉONTOLOGIE, VOL. $45, \mathrm{~N}^{\circ} 4$ 
plus par l'apparition de Discoaster bifax. Tout cela semble indiquer la zone NP $15 \mathrm{c}$ et la partie sommitale de la CP 13c. Les premiers exemplaires de Reticulofenestra umbilica typiques (longueur $>15 \mu \mathrm{m}$; Pl. 1, fig. 3) et de Cribrocentrum reticulatum se rencontrent dans l'échantillon sus-jacent $Z$. La coexistence de ces formes, associée à la présence de Blackites gladius (Pl. 1, fig. 1 et 2 ) et à la disparition de Lophodolithus acutus, permet une attribution à la partie sommitale de NP 15e et la partie basale de la CP 14a. Toutes les associations en provenance de la petite falaise $0(0 \mathrm{~g}, 0 \mathrm{a}$ à $0 \mathrm{~d})$ se ressemblent de très près. Elles contiennent Helicosphaera salebrosa (Pl. 1, fig. 10), H. papillata (Pl. 1, fig. 5), Chiasmolithus solitus, S. furcatolithoides, S. spiniger (Pl. 1, fig. 27), et quelques rares $B$. gladius. La présence de $B$. gladius permettrait par définition l'inclusion de ces associations dans la zone NP 15. Cependant, vu leur rareté, nous croyons que ces spécimens sont remaniés, et que, par la présence de $H$. salebrosa et $S$. spiniger, cette association se rattache plutôt à la zone NP 16. Les échantillons 5 et 6 récoltés dans le lit du petit ruisseau au nord de la ferme Luscly (Fig. 2) sont marqués par les apparitions de Dictyococcites bisectus, de Bramletteius serraculoides, de Sphenolithus obtusus et de Clathrolithus spinosus. La présence de ces formes, associée à l'absence de $C$. solitus, suggère la partie basale de la zone NP 17.

Les assemblages des premières couches glauconieuses dans l'escarpement II (II b, II c), une dizaine de mètres en amont de la cascade de Bagats, sont marquées par les apparitions de Corannulus germanicus (PI. 1, fig. 15), Hayella situliformis, Helicosphaera bramlettei, H. compacta (Pl. 1, fig. 21), H. reticulata et $H$. wilcoxonii (PI. 1, fig. 20), et par la fréquence de $R$. umbilica et $C$. reticulatum (Pl. 1, fig. 11). Une telle association, marquée par la présence de Hayella situliformis, Helicosphaera reticulata et $\mathbf{H}$. wilcoxonii et l'absence de Chiasmolithus oamaruensis, semble indiquer la partie supérieure de la zone NP 17 (Perch-Nielsen, 1985; Aubry, 1992). Les rares exemplaires de Chiasmolithus solitus sont interprétés comme remaniés. Toutefois, notons que dans le Bassin de la Mer du Nord D. bisectus apparaît déjà dans la zone NP 16 (Aubry, 1986), tandis que dans la région Crimée-Caucase les apparitions de $H$. bramlettei, $H$. compacta et $D$. bisectus précèdent la disparition de $C$. solitus. Dans le cas où il ne s'agit pas de remaniement, celles-ci se situent donc au sein de la zone NP 16 (Shcherbinina, 2000).

Les premières couches en aval de la cascade sont celles qui se trouvent au pied de la coupe I. Ces niveaux glauconieux (échantillon Ia) sont caractérisés par la présence de Chiasmolithus oamaruensis (Pl. 1, fig. 17 et 18) marqueur de la base de la zone NP 18. Tous les échantillons de la coupe I contiennent également $C$. grandis (Pl.1, fig. 25 et 26). D'après les zonations standard figurant dans Berggren et al. (1995) ces deux espèces ne coexistent pas, mais sont séparées par un intervalle de temps de cent mille ans (disparition de $C$. grandis à 37.1 Ma ; apparition de $C$. oamaruensis à $37.0 \mathrm{Ma}$ ). Toutefois, les observations faites dans la région-type du Priabonien (Barbin, 1988), ainsi que celles dans la région de Biarritz (Mathelin, 1988 ; Mathelin et Sztràkos, 1993) contredisent cette constatation et montrent que $C$. grandis persiste encore dans les zones NP 18 et NP 19-20. Nos propres recherches en Aquitaine suggèrent que la plupart de ces observations correspondent à des remaniements (la présence d'un seul ou quelques exemplaires assez souvent abîmés est interprétée ainsi). Par contre, dans la coupe du Ruisseau de Lespontes cette espèce reste assez fréquente dans les niveaux appartenant à la partie basale de la zone NP 18. Cette fréquence nous incite à croire que $C$. grandis a pu survivre la limite NP17/NP18 en Aquitaine, jusqu'au début du Biochrone NP 18. L'espèce Clathrolithus spinosus (Pl. 1, fig. 23) est assez fréquente dans l'échantillon Ia.

Une association semblable à celles de la coupe $\mathrm{I}$, a été identifiée à la base de la coupe IV (IV a), $20 \mathrm{~m}$ en aval de la cascade de Bagats. Elle s'en distingue par l'absence de C. grandis et par l'apparition de Lithostromation deflandrei (IV d ; Pl. 1, fig. 19). Les couches de la coupe IV appartiennent donc à une partie plus élevée de la zone NP 18. En peu plus en aval affleurent des niveaux grésifiés glauconieux de la coupe V. Ils sont séparés des couches marneuses de la coupe IV par une faille. La coexistence de $C$. grandis et $C$. oamaruensis dans la coupe $\mathrm{V}$ démontre sa position à la base de la zone NP 18 , inférieure à celle de la coupe IV. Nous n'avons pas observé Isthmolithus recurvus dans la suite de la coupe du Ruisseau de Lespontes, ce qui suggère que les couches en aval de la cascade de Bagats jusqu'au sommet de la grande falaise E se rattachent à la zone NP 18. Plusieurs autres événements ont été enregistrés au sein de cette zone, parmi lesquels : la disparition de Neococcolithes minutus (PI. 1, fig. 16), la forte diminution de Cribrocentrum reticulatum (de 4 exemplaires sur 10 champs de vue à moins d'un exemplaire sur 20 champs de vue à grandissement $1000 x$ ) et les apparitions de Naninfula sp. (forme rare à épine pointue, assez mince, nettement différente de Naninfula deflandrei; cette dernière espèce est présente dans l'échantillons $\mathrm{V}$ et $\mathrm{W}$ ) et de Cyclicargolithus neogammation (espèce considérée valable, vu le diamètre $(9 \mu \mathrm{m})$ nettement plus grand que celui des types de $C$. floridanus : 4 à $5 \mu \mathrm{m}$ ) entre les échantillons 
V et U, et la présence de Sphenolithus tribulosus au sommet de la coupe (échantillons B et E 9). Actuellement il est difficile de juger si ces événements ont une valeur biostratigraphique locale significative, faute de l'absence de coupes contemporaines continues en Aquitaine.

\section{LES FORAMINIFÈRES}

Dans sa monographie sur les foraminifères éocènes du Bassin de l'Adour Sztràkos (2000) a proposé une nouvelle biozonation de foraminifères planctoniques, plus fine que les biozonations classiques élaborées en région tropicale et pyrénéenne. Cette nouvelle biozonation, spécifique à l'Aquitaine, comprend seize unités (EPA 1 à 16). Elle a pu être mise en corrélation avec les zonations classiques (parmi lesquelles les zones $\mathrm{P}$ de Berggren et al., 1995), vu le grand nombre d'espèces-guides communes.

La partie la plus ancienne de la coupe ayant fourni des foraminifères est représentée par les échantillons DB 412 et 413, imprécisément localisés par Boulanger (1968). Dans les associations de foraminifères planctoniques (Tableau 1), composées parmi d'autres de Turborotalia cerroazulensis frontosa, T. cerroazulensis possagnoensis, Guembelitrioides higginsi, Acarinina broedermanni, Globigerinatheka mexicana barri, on observe encore quelques Morozovella aragonensis, marquant le sommet de la zone à Globigerinatheka subconglobata (P 11). La présence de Nuttallides truempyi dans la faune de foraminifères benthiques (qui comportent jusqu'à 150 espèces par échantillon) indique une paléoprofondeur de plus de $500 \mathrm{~m}$ (van Morkhoven et al., 1986).

Les échantillons DB 411, Y, Z et $0 \mathrm{~g}$ à b (Fig. 2) appartiennent probablement à la partie inférieure de la zone à Morozovella lehneri (P 12, zone EPA 10), à cause de l'absence de $M$. aragonensis et la présence de Acarinina broedermanni. Des remaniements du Crétacé, Paléocène (Globanomalina pseudomenardii) et Lutétien basal (Turborotalia griffinae) apparaissent occasionnellement. A. broedermanni n'est plus représenté dans les échantillons Oc et Od. Ceuxci contiennent les derniers $T$. cerroazulensis possagnoensis et Guembelitrioides higginsi, appartenant vraisemblablement à la partie supérieure de la zone à Morozovella lehneri (P 12, zone EPA 11). La coupe II est caractérisée par la présence de rares Globigerinatheka index tropicalis, T. cerroazulensis pomero$l i, T$. cerroazulensis cerroazulensis et Truncorotaloides rohri. La coexistence de ces formes permet une attribution à la zone EPA 13 et la partie médiane de la zone P 14. Notons également que des foraminifères remaniés sont assez rares dans cette coupe, mais des remaniements du Trias (cristaux de quartz bipiramidés) étaient présents (éch. II c). La faune de foraminifères benthiques est composée de 63 à 86 espèces où Uvigerina acutocostata, U. eocaena, Globobulimina pyrula, Sporobulimina eocaena, Bolivina vaceki, B. semistriata, Riminopsis rotulus, Heterolepa eocaena et Gyroidinoides girardanus dominent.

L'échantillon I a est avant tout marqué par le grand nombre de formes remaniées $(T$. cerroazulensis possagnoensis, P. wilcoxensis, Guembelitrioides higginsi, etc.). Les 74 espèces de foraminifères benthiques caractérisent le milieu circalittoral à épibathyal supérieur. Les échantillons I b-e contiennent également des remaniements de foraminifères planctoniques provenant des marnes lutétiennes sous-jacentes, comme G. higginsi. Les foraminifères planctoniques autochtones indiquent la partie supérieure de la zone à Truncorotaloides rohri $(\mathrm{P}$ 14, zone EPA 13), à cause de la coexistence des formes épineuses de l'Eocène moyen s. l. (Acarinina bullbrooki, A. spinuloinflata, Truncorotaloides rohri, T. topilensis), de Turborotalia cerroazulensis cerroazulensis et Globigerinatheka index tropicalis, dont l'apparition marque la base de la zone EPA 13. La faune de foraminifères benthiques est riche d'une centaine d'espèces par échantillon. Elle caractérise un milieu circalittoral d'une profondeur entre 100 et $200 \mathrm{~m}$. Elle est dominée par Uvigerina acutocostata, U. eocaena, Cibicidoides alleni, C. bionus, Riminopsis rotulus et Gyroidinoides girardanus. Les nummulites déplacées, mal conservées, sont présentes sporadiquement.

Les foraminifères des échantillons $\mathrm{c}$ et e ont été étudiés dans la coupe IV. Ils contiennent encore de rares globorotalidés épineux : Morozovella spinulosa, Acarinina spinuloinflata, A. bullbrooki, Truncorotaloides rohri et $T$. topilensis, sans que l'on puisse savoir s'il s'agit d'éléments en place ou de remaniements. Globigerinatheka semiinvoluta est absente. Dans le cas où il ne s'agirait pas de remaniements, ces associations pourraient appartenir aux zones P 14 ou EPA 13. L'association de foraminifères benthiques de ce niveau est diversifiée (73 à 96 espèces).

La partie sus-jacente des Marnes de Brihande, représentée par les échantillons V, U, DB 414, 416, et 491 est riche en foraminifères : 90 à 130 espèces benthiques et 10 à 15 planctoniques. Globigerinatheka semiinvoluta est fréquente dans les échantillons DB 414 et 416, rare dans celui de U et DB 491, et réfère aux zones $\mathrm{P} 15$ et EPA 15. Par la présence de globorotalidés épineux et l'absence de Globigerinatheka semiinvoluta l'échantillon $\mathrm{V}$ semble se ratta- 


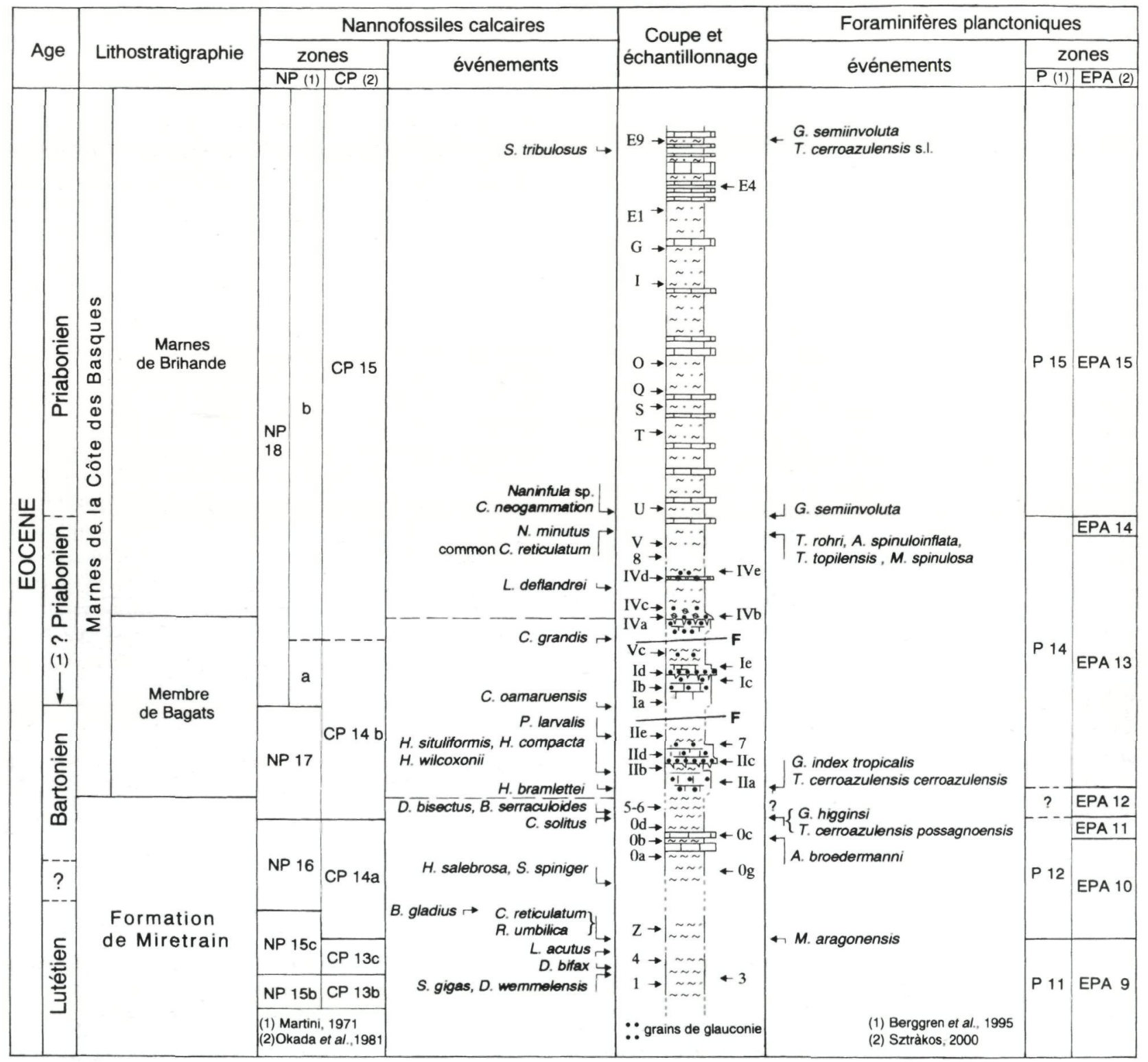

FIG. 4. Les principaux événements à nannofossiles calcaires et foraminifères planctoniques dans l'Eocène moyen et supérieur de la coupe du Ruisseau de Lespontes. (1) limite bartonienne/priabonienne sensu Berggren et al. (1995), basée sur l'apparition du nannofossile $C$. oamaruensis. The major calcareous nannofossil and planktonic foraminiferal events in the Middle and Upper Eocene of the Lespontes River section.

(1) Bartonian/Priabonian boundary sensu Berggren et al. (1995) based on the appearance of the nannofossil C. oamaruensis.

cher à la partie sommitale de la zone EPA 13. Le nombre d'espèces de foraminifères benthiques diminue progressivement en montant dans la coupe, de 80 à 60 , entre les échantillons $\mathrm{T}$ et $\mathrm{A}$. En même temps, Siphonodosaria crassisepta devient prédomi- nante, suivie peu après par Ceratocancris crassus, Hoeglundina elegans, Uvigerina eocaena, Planulina ruizi, Cibicidoides alleni, Heterolepa eocaena, Alabamina wilcoxensis et Almaena epistominoides. Quelques échantillons sont riches en grands forami- 
TABLEAU 1. - Répartition des foraminifères planctoniques dans la coupe du Ruisseau de Lespontes à Saint-Lon-les-Mines. Abréviations : $\mathbf{r}-$ rare ; $\mathrm{c}-$ commun ; f - fréquent ; R remaniement (pour la localisation de Ex, y, z voir Fig. 2, et de E1-E9 voir Fig. 4 ; la localisation des échantillons DB, en provenance de l'ancienne collection D. Boulanger, est incertaine). Range chart of the planktonic foraminifera in the Lespontes River section. Abbreviations $: r$-rare ; $c$-common; $f$-frequent $; R-$ reworked (for the location of Ex, $y, z$ see Fig. 2 , and of E1-E9 see Fig. 4 ; the location of the DB samples, taken from the collection D. Boulanger, is uncertain).

\begin{tabular}{|c|c|c|c|c|c|c|c|c|c|c|c|c|c|c|c|c|c|c|c|c|c|c|c|c|c|c|c|c|c|c|c|c|c|c|}
\hline \multirow{2}{*}{$\begin{array}{r}\text { BIOZONES } \\
\text { FORMATIONS } \\
\text { MFIDORCS }\end{array}$} & G. su & con. & \multicolumn{7}{|c|}{ M. lehneri } & \multicolumn{14}{|c|}{ T. rohri supérieure } & \multicolumn{11}{|c|}{ G. semiinvoluta } \\
\hline & \multicolumn{9}{|c|}{ Formation de Miretrain } & \multicolumn{25}{|c|}{ Marnes de la Côte des Basques } \\
\hline MEMBRES & & & & & & & & & & \multicolumn{11}{|c|}{ Membre de Bagats } & & & & & & & Marne: & s de B & Brihan & & & & & \\
\hline & & $\mathrm{DB}$ & & & & & & & & & & & & & & & & & & & & & & & & & & $\mathrm{DB}$ & & & & & & \\
\hline ECHANTILLONS & 412 & 413 & 411 & Y & $\mathrm{z}$ & 0.9 & $0 . \mathrm{b}$ & & $0 . d$ & 11.a & J11.6 & II.c & III.d & & L.a & & & 1.d. & & V.c & N.c. & N.e & |v & U & 414 & 491 & 415 & 489 & 490 & 492 & 416 & & D-A & $E x, Y ; 1.9$ \\
\hline Laterostomella martini (PIJPERS, 1933) & & & & $r$ & $\mathrm{r}$ & & & $\mathrm{r}$ & & & $\mathrm{r}$ & & & & $r$ & & & & & & $r$ & $r$ & & & $r$ & $r$ & & $r$ & & - & $\square$ & $\mathrm{r}$ & $r$ & $r$ \\
\hline Planorotalites pseudoscitulus (GLAESSNER, 1937) & & & & & & $r$ & & & $r$ & & $r$ & & $r$ & & - & & $r$ & & & 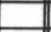 & & & & & 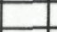 & & & & & & & & & \\
\hline Turborotalia bolivariana (PETTERS, 1954) & & $\mathrm{r}$ & & & c & - & c & 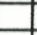 & $\mathrm{r}$ & + & +1 & $\rightarrow$ & 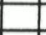 & & & & 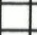 & & & & & & $\square$ & | & & & & & & & & & & \\
\hline T. cerroazulensis cerroazulensis (COLE, 1928) & & & & & & & & & & $r$ & $\mathrm{r}$ & $\mathrm{r}$ & $r$ & . & & c & $\mathrm{r}$ & $\mathrm{r}$ & c & c & c & $\mathbf{r}$ & c & 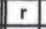 & & c & & c & $\mathrm{c}$ & $\mathrm{c}$ & $r$ & $r$ & $r$ & $r$ \\
\hline T. cerroazulensis cocoaensis (CUSHMAN, 1928) & & & & & & & & & & & & & & & & & & & & & & & & & & & & $r$ & $r$ & $r$ & & $r$ & $\mathrm{r}$ & $r$ \\
\hline T. cerroazulensis frontosa (SUBBOTINA, 1953) & c & $t$ & c & c & $\mathrm{r}$ & $r$ & $r$ & $\mathrm{r}$ & $\begin{array}{rr} \\
\end{array}$ & 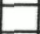 & & & & & $r$ & & $r$ & $r$ & & & & & & 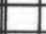 & & & & & & & & & & \\
\hline T. cerroazulensis pomeroli (TOUMARKINE et BOLLLI, 1970) & & & of & & & & & & & & $\mathrm{r}$ & & $r$ & c & $r$ & $r$ & & $\mathrm{r}$ & $r$ & $\mathbf{r}$ & c & $r$ & o & 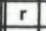 & $\circ$ & $r$ & $\mathbf{r}$ & & & & $r$ & c & & \\
\hline T. cerroazulensis possagnoeensis (TOUMARKINE et BOLLI, 1970) & $r$ & o & $\mathbf{r}$ & c & c & $c$ & c & c & 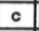 & & & & & & $\mathbf{A}$ & & R & $\mathrm{R}$ & & & & & & & & & & & & & & & & \\
\hline T. griffinae (BLOW, 1979) & $\bar{R}$ & & $\mathbf{R}$ & $\mathbf{R}$ & & & & & & & & & & & & & & & $\mathrm{R}$ & & $\mathbf{A}$ & & & & & & & & & & & & & \\
\hline Acarinina broedermanni (CUSHMAN et BERMUDEZ, 1949) & $r$ & c & c & & $\mathrm{r}$ & & $r$ & & & & & & & L & & & $\square$ & & & & & & & & & & & & & & & & & \\
\hline A. bulibrooki (BOLLL, 1957) & $t$ & c & $\circ$ & $r$ & $r$ & c & c & c & & $r$ & & $r$ & & r & $\mathrm{r}$ & & $r$ & $r$ & $r$ & $r$ & & & & & & & & & & & & & & \\
\hline A.? medizzai (TOUMARKINE et BOLLI, 1975) & & & & & & & & & & & & & & & 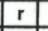 & & & & & & & & & & & & & & & & & & & \\
\hline A. spinuloinflata (BANDY, 1949) & & $f$ & $f$ & c| & $f$ & 1 & $f$ & $\mathrm{f}$ & $\mathrm{f}$ & 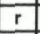 & $\mathrm{r}$ & \begin{tabular}{|r|r} 
\\
\end{tabular} & 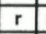 & r & & $\mathrm{r}$ & $\mathrm{r}$ & & c & $\mathrm{r}$ & & $r$ & $r$ & & & & & & & & & & & \\
\hline Morozovella aragonensis (NUTTALL, 1930) & $r$ & $r$ & & & & & & & & & & & & & & & & & & & & & & & & & & & & & & & & \\
\hline M. spinulosa (CUSHMAN, 1927) & & & & $r$ & $\mathrm{r}$ & $\mathrm{r}$ & $r$ & $r$ & c & $r$ & $\mathrm{r}$ & & $r \mid$ & & $r$ & $\mathrm{r}$ & $r$ & $\mathrm{r}$ & $r$ & $\mathrm{r}$ & & $r$ & $r$ & & & & & & & & & & & \\
\hline Truncorotaloides rohri BROONNIMANN et BERMUDEZ, 1953 & $r$ & $r$ & $r$ & $r$ & $\mathrm{r}$ & $\mathrm{r}$ & & & & $r$ & c & & $\mathrm{r}$ & & $r$ & $\mathrm{r}$ & $r$ & $\mathrm{r}$ & $r$ & $\mathrm{r}$ & $r$ & $r$ & $r$ & & & & & & & & & & & \\
\hline T. topilensis (CUSHMAN, 1925) & $r$ & & & $r$ & $\mathrm{r}$ & $r$ & $r$ & $r$ & $\mathrm{r}$ & $r$ & $r$ & $r$ & $\mathrm{r}$ & $r$ & $\mathrm{r} \mid$ & $\mathrm{r}$ & $\mathrm{r}$ & $\mathrm{r}$ & $\mathrm{r}$ & $r$ & & & $r$ & & & & & & & & & & & \\
\hline Catapsydrax dissimilis (CUSHMAN et BERMUDEZ, 1937) & & & & & & & & & & & & & & & & & & & & & & & & & & $\mathrm{r}$ & & $\circ$ & & $\mathrm{r}$ & & $\mathrm{r}$ & & $r$ \\
\hline Globorotaloides carcosellensis TOUMARKINE et BOLLI, 1975 & & $r$ & & & $\mathrm{r}$ & $r$ & $r$ & & $r$ & & c & & c & & $\mathrm{r}$ & $\mathrm{r}$ & $r$ & $\mathrm{r}$ & c| & $\mathrm{r}$ & $r$ & $r$ & $r$ & & & & $r$ & & & & & & & \\
\hline G. suteri BOLLI, 1957 & & & & & & & & & & & & & & & & & & & & & & & & & & $r$ & & $r$ & & & $r$ & & & \\
\hline Guembelitrioides higginsi (BOLLI, 1957) & $r$ & $f$ & $\mathrm{c}$ & c) & $\mathrm{r}$ & $r$ & $r$ & $r$ & $r$ & $\mathrm{~A}$ & $\mathrm{R}$ & & 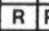 & $\mathrm{B}$ & 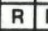 & $\mathrm{R}$ & B & $\mathrm{R}$ & $\mathrm{R}$ & & $\mathbf{R}$ & $\mathbf{R}$ & & & & & & & & & & & & \\
\hline Subbotina eocaena eocaena (GUMBEL, 1870) & $t$ & $\mathrm{c}$ & $t$ & 1 & c & c & c & c & c & c & c & $r$ & 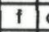 & c] & \begin{tabular}{|c|c|c|c|c|}
$c$ & 1 \\
\end{tabular} & c & c| & c & c) & $f$ & $t$ & $t$ & c & c & c & c & c & $t$ & $\mathrm{c}$ & $r$ & $t$ & $r$ & $r$ & $r$ \\
\hline S. eocaena cryptomphala (GLAESSNER, 1937) & & & & & & $r$ & & & & c & & $\mathrm{r}$ & & r & c & $\mathrm{r}$ & & c & c & & c & c & \begin{tabular}{r||l} 
\\
\end{tabular} & 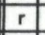 & c & $r$ & $r$ & & & & & $r$ & & \\
\hline S. hagni (GOHRBANDT, 1967) & c & c & 0 & $r$ & - & c & c & 1 & 1 & & $r$ & & & & & & & & & $\mathrm{r}$ & & & & & & & & & & & & \begin{tabular}{|r} 
\\
\end{tabular} & & \\
\hline S. inaequispira (SUBBOTINA, 1953) & & & $r$ & & & & & & & & & $\mathrm{R}$ & 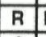 & R & 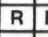 & R & R & $\mathrm{R}$ & R & & $\mathbf{R}$ & $\mathbf{R}$ & & & & & & . & & & & & & \\
\hline S. linaperta linaperta (FINLAY, 1939) & $f$ & & $\mathrm{c}$ & & c & & 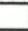 & & 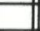 & $r$ & 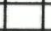 & $\begin{array}{rr}r & 2 \\
\end{array}$ & 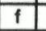 & $\mathrm{r}$ & $r \mid$ & $\mathrm{r}$ & of & $r$ & c & c & c & $\mathrm{c}$ & \begin{tabular}{|c|c|}
$c$ \\
\end{tabular} & c & $r$ & & $r$ & c & $\circ$ & & \begin{tabular}{|c|ll}
$c$ & \\
\end{tabular} & $r$ & $r$ & $r$ \\
\hline S. linaperta utilisindex (JENKINS et ORR, 1973) & & & & & & & & & & & & & & & & & & & & & & & & & $r$ & $r$ & & & & & & & & \\
\hline S. tripartita (KOCH, 1926) & & & & & & & & & & & & & & & & & & & $\mathrm{r}$ & $\mathrm{r}$ & & & \begin{tabular}{|r|r} 
& -1 \\
\end{tabular} & & & & $r$ & c & & & & $\mathrm{r}$ & & \\
\hline Clavigerinella eocanica (NUTTALL, 1928) & & & 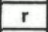 & & & & & & & & & & & & & & & & & & $\mathbf{R}$ & & & & & & & & & & & & & \\
\hline Psoudohastigerina micra (COLE, 1927) & $r$ & $r$ & $r$ & c & $\mathrm{r}$ & $r$ & $r$ & c & $r$ & $r$ & c| & & $r$ & & $r \mid$ & $\mathrm{r}$ & $r$ & $\mathrm{r}$ & $r$ & & $r$ & $c$ & $r$ & & $r$ & $r$ & $r$ & & $\mathrm{r}$ & & $\mathrm{r}$ & $r$ & $r$ & $r$ \\
\hline P. wilcoxensis (CUSHMAN et PONTON, 1932) & & & & c & $r$ & & & - & $r$ & & & & & & 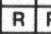 & R & & & A & & & & & & & & & & & & & & & \\
\hline Hantkenina alabamensis CUSHMAN, 1925 & & & & & & & & & & & & $r$ & & & & & & & $\mathrm{r}$ & & & & & & & & $r$ & & & & & & & \\
\hline H. dumblei WEINZIERL et APPLIN, 1929 & & $r$ & & $r$ & $\mathrm{r}$ & $r$ & $r$ & $\mathrm{r}$ & $r$ & & & & & & & & & & & & 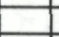 & & - & & & & & & & & & & & \\
\hline H. longispina CUSHMAN, 1925 & & & & & & & & & & & & & & & & & & & & & & & & & & & & & & & $r$ & & & \\
\hline Globigerina of. ouachitaensis gnaucki BLOW et BANNER, 1962 & & & & & & & $r$ & & $r$ & & $r$ & & & r & & & & & & & $\mathrm{r}$ & & & & & & & & & & & & & \\
\hline G. ouachitaensis ouachitaensis HOWE et WALLACE, 1932 & & & & & & & & & & & & & & & & & & & & & & & & & & of & & & & & of & & & \\
\hline G. praebulloides BLOW, 1959 & & & & & & & & & & & & & & & & & & & & & $?$ & & & & & $\mathbf{r}$ & & & & & & $\mathrm{r}$ & & $\mathrm{r}$ \\
\hline Globigerinatheka index index (FINLAY, 1939) & & ef & & & $\mathrm{r}$ & & c & & & c & c & $\mathrm{r}$ & 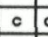 & ct & \begin{tabular}{|l|ll}
$f$ &
\end{tabular} & c & cf & & c & $\mathrm{r}$ & c & $c$ & of & 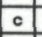 & & $r$ & & & - & $\mathrm{r}$ & $\mathrm{c}$ & $\mathrm{r}$ & $r$ & $\mathrm{r}$ \\
\hline G. index rubriformis (SUBBOTINA, 1953) & & & & & & & & & & & & & & & & & & & & $r$ & & & & & & & & & & & & & & $\mathrm{r}$ \\
\hline G. index tropicalis (BLOW ot BANNER, 1962) & & & & & & & & & & $\mathrm{r}$ & a & & $r \mid$ & r & & c| & & $\mathrm{r}$ & if & $\mathrm{c}$ & $f$ & $f$ & 0 & \begin{tabular}{|c|cc}
$c$ & 2 & 0 \\
\end{tabular} & $r$ & $\mathrm{c}$ & c & $r$ & $\mathrm{r}$ & & $\begin{array}{ccc}\mathrm{c} \\
\end{array}$ & $r$ & & $r$ \\
\hline G. mexicana barri BRONNIMANN, 1952 & & $r$ & & & & $r$ & & $\mathrm{r}$ & $r$ & & $\mathrm{r}$ & $r$ & & T & & - & & $r$ & & & & & & & & & & & & & & & & \\
\hline G. mexicana mexicana (CUSHMAN, 1925) & & & & $f$ & $\begin{array}{ll} \\
\end{array}$ & $f$ & $t$ & c| & c & c & & $\mathrm{r}$ & & . & \begin{tabular}{|c|ccccc}
$r$ & $c$ & $r$
\end{tabular} & c & $r$ & c & c & $r$ & $\mathbf{r}$ & $r$ & . & 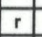 & $r$ & $r$ & $r$ & $r$ & $r$ & $r$ & & $r$ & $r$ & $r$ \\
\hline G. semiinvoluta (KEIJZER, 1945) & & & & & & & & & & & & & & & & & & & & & & & & \begin{tabular}{r|r}
$r$ \\
\end{tabular} & $\mathrm{f}$ & $r$ & $f$ & $t$ & c & & $\mathrm{c}$ & $r$ & & $r(E y)$ \\
\hline G. senni (BECKMANN, 1954) & & $r$ & & & $r$ & & $r$ & $r$ & & & & & & & & & & & & & & & & & & & & & & & & & & \\
\hline G. subconglobata (SHUTSKAYA, 1958) & & & & & & $r$ & $r$ & & $r$ & & & & & & 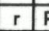 & 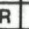 & & & & & & & & & & & & & & & & & & \\
\hline
\end{tabular}


nifères déplacés (B, D, E1): Operculina alpina, Nummulites variolarius, Discocyclina sp.

En montant dans la falaise $\mathrm{E}$ la faune des foraminifères continue à s'appauvrir, de 60 à 30 espèces benthiques et de 6 à 2 planctoniques par échantillon. Cette association planctonique est proche de celle de la carrière de Lassalasse, près de Siest (DB 183, Sztràkos et al., 1998, p. 88). Les espèces benthiques citées ci-dessus continuent à dominer. La présence de Globigerinatheka mexicana dans les échantillons E1 et E4 montre que la falaise appartient encore à la zone à Globigerinatheka semiinvoluta (cette espèce disparaît avant le marqueur de la biozone d'après Toumarkine et Luterbacher, 1985).

Les foraminifères planctoniques de la coupe du Ruisseau de Lespontes sont repertoriés dans le Tableau 1. La faune de foraminifères benthiques comporte plusieurs centaines d'espèces. Leur répartition stratigraphique est conforme au tableau publié par Sztràkos (2000).

\section{CALIBRATION DES ZONATIONS UTILISÉES}

En comparant les diverses zonations à foraminifères planctoniques Sztràkos (2000) et Sztràkos et Castelltort (2001) ont démontré que dans l'Eocène d'Aquitaine les apparitions et/ou les disparitions de certaines espèces sont nettement décalées part rapport à celles observées en région tropicale. Limitonsnous à l'apparition de Globigerinatheka semiinvoluta, qui définit la base de la zone standard P 15. En région pyrénéenne, y compris la coupe de Biarritz (Mathelin, 1988), celle-ci est précédée par la disparition de Truncorotaloides rohri. Par contre, en région tropicale c'est l'apparition de G. semiinvoluta qui la précède. Dans la coupe du Ruisseau de Lespontes ces deux espèces ne coexistent non plus (voir Tableau 1, fig. 4), confirmant ainsi les observations faites en région pyrénéenne.

Notre étude permet également de calibrer les zonations à foraminifères planctoniques à celles à nannofossiles calcaires et de les comparer au modèle biostratigraphique standard de Berggren et al. (1995). Ainsi, en Aquitaine, l'apparition de Chiasmolithus oamaruensis, par définition marquant la base de la zone NP 18, est nettement antérieure à l'apparition de $G$. semiinvoluta. Elle est également antérieure à la disparition de Truncorotaloides rohri et légèrement postérieure à l'apparition de $G$. index tropicalis, affirmant sa position au sein de la zone
EPA 13 et dans la partie médiane de la P 14 (Fig. 4). Ce problème a été discuté dans la publication de Sztràkos, 2000 et Sztràkos et Castelltort, 2001. En ce point nos données sont en désaccord avec le modèle standard de Berggren et al., montrant que cet événement est positionné au milieu de la zone P I5. Ceci a également des répercussions pour la limite bartonienne/priabonienne. Actuellement, en absence d'un point repère ou «Global Stratotype Section and Point (GSSP)«, il est généralement admis que I'apparition de $C$. oamaruensis représente le critère pour la définition de cette limite (Berggren et al., 1995, p. 197). Si l'on accepte cette proposition, cette limite se placerait au sein des Marnes de Bagats en Aquitaine, dans la zone P 14, bien avant la limite admise à l'aide des foraminifères planctoniques par Berggren et al.

\section{CONCLUSIONS}

L'étude détaillée de divers affleurements le long du Ruisseau de Lespontes et ses affluents à SaintLon-les-Mines permet de reconstituer la succession des corps sédimentaires d'âge éocène moyen et supérieur dans le Synclinal du Bassecq et de les dater à partir des associations à nannofossiles calcaires (zones NP) et à foraminifères planctoniques (zones locales EPA et zones standard P). Les niveaux les plus bas de la coupe se situent entre la ferme Lartigue, $0.5 \mathrm{~km}$ à l'est de la ferme Luscly, et l'usine de Bagats. Ce sont des marnes bleuâtres à faciès hémipélagique, légèrement inclinées vers le nord. Ils représentent les Marnes de Miretrain. L'ensemble des foraminifères benthiques indique un milieu de dépôt bathyal. Ces marnes appartiennent aux zones à nannofossiles NP 15b, NP 15c, NP 16 et base de la NP 17 et aux zones à foraminifères planctoniques locales EPA 9 à EPA 11 (les échantillons 5 et 6, dont les foraminifères n'ont pas encore été étudiés, pourraient appartenir à la EPA 12 et ? P 13), qui sont corrélatives à l'intervalle milieu P 11 - sommet P 12 de la zonation standard de Berggren et al. Ceci suggère un âge lutétien supérieur et bartonien inférieur pour ces niveaux.

Le contact avec les Marnes de Bagats sus-jacentes n'a pas été observé. Cette unité, membre de la Formation des Marnes de la Côte des Basques et nouvellement introduite dans la présente note, affleure juste à l'ouest de l'usine de Bagats. Il s'agit d'un complex de marnes gréseuses et de grès, très glauconifères et de niveaux marneux, peu glauconieux et moins puissants. Cet ensemble est fortement tectoni- 
sé. La partie exposée comprend la partie supérieure de la NP 17 et la base de la NP 18 et appartient à la zone EPA 13 et la partie médiane de la P14. L'apparition de Chiasmolithus oamaruensis, jusqu'au nouvel ordre le critère généralement admis pour la définition de la limite bartonienne/priabonienne, se situe donc au sein des Marnes de Bagats. La faune de foraminifères benthiques caractérise un milieu circalittoral d'une profondeur entre 100 et $200 \mathrm{~m}$. Ces Marnes de Bagats appartiennent à un prisme de bas niveau, comparable au Complexe chaotique d'Ilbarritz et de l'Horizon à lumachelles de la Côte des Basques, à la base de la séquence E 15 de Sztràkos et al. (1998). L'abondance de la glauconie à gros grains et des restes de poissons, associée à la présence des surfaces durcies sont les indices d'une sédimentation condensée. L'alternance des niveaux gréseux à glauconie et des niveaux marneux semble être provoquée par la capture périodique des sediments grossiers glauconieux en amont, en relation avec les mouvements tectoniques et du diapirisme dans la proximité de l'anticlinal de Saint-Lon-les-Mines.

Le contact avec les Marnes de Brihande finement sableuses a été recensé à la base de la coupe IV, à environ $40 \mathrm{~m}$ en aval de la cascade de Bagats. Il s'agit d'une surface bioturbée, recouverte par une couche fossilifère à éléments grossiers, parmi lesquels otolithes de poissons et dents de requins. Cette surface est interprêtée comme limite de la séquence de dépôt $\mathrm{E}$ 16 de Sztràkos et al. (1998).

La base des Marnes de Brihande est attribuable à la partie inférieure, mais pas basale, de la zone NP 18 et à la partie sommitale de la EPA 13. Les premières Globigerinatheka semiinvoluta marquant la base de la EPA 15, ont été recensées dans l'échantillon $\mathrm{U}$ à $50 \mathrm{~m}$ au $\mathrm{N}$ du tunnel sous l'usine de Bagats (verticalement environ $10 \mathrm{~m}$ au-dessus de la base des Marnes de Brihande). En descendant le cours du Lespontes les niveaux stratigraphiques des Marnes de Brihande se succèdent progressivement sous le même faciès. La faune des foraminifères continue à s'appauvrir, de 60 à 30 espèces benthiques et de 6 à 2 planctoniques par échantillon. Vers le sommet de la coupe des calcaires viennent s'intercaler dans la série marneuse. Ces alternances marquent la transition vers les Calcaires de Siest. L'absence de Isthmolithus recurvus suggère que la totalité des Marnes de Brihande dans la coupe du Lespontes se rattache à la zone NP 18. Mais comme cette espèce est rare en Aquitaine, cela reste assez spéculatif. La présence de Globigerinatheka mexicana dans les échantillons E 1 et $\mathrm{E} 4$ montre que la falaise au sommet de la coupe appartient encore à la zone à G. semiinvoluta.
L'intégration des données stratigraphiques de la coupe du Ruisseau de Lespontes confirme que l'apparition de G. semiinvoluta est hétérochrone et nettement plus tardive en région pyrénéenne. Elle y est postérieure à l'apparition de Chiasmolithus oamaruensis, actuellement admise comme critère pour la définition de la limite bartonienne/priabonienne.

\section{REMERCIEMENTS}

Nous tenons à remercier B. Cahuzac (Université de Bordeaux) pour l'utilisation de sa photothèque et H. De Potter et J. Cilis (IRSNB, Bruxelles) pour le soin qu'ils ont mis à la mise au point de nos figures et la réalisation de la planche. La présente note est une contribution au projet de recherche $\mathrm{n}^{\circ} \mathrm{G} .0093 .01$ sur la transition éocène - oligocène, financé par le FWO (Fonds de la Recherche Scientifique) - Vlaanderen.

\section{BIBLIOGRAPHIE}

Aubry M.-P. (1986) : Paleogene calcareous nannoplankton biostratigraphy of northwestern Europe. Palaegeogr., Palaeoclimatol., Palaeoecol., vol. 55, p. 267-334.

Aubry M.-P. (1992) : Late Paleogene calcareous nannoplankton evolution : A tale of climatic deterioration. In Prothero, D.R. et Berggren, W.A. (eds) : Eocene-Oligocene Climatic and Biotic Evolution, Princeton University Press, Princeton, p. 272 309.

Aubry M.-P. (1999) : Handbook of Cenozoic Calcareous Nannoplankton. Book 5 : Heliolithae (Zygoliths and Rhabdoliths). Micropaleontology handbook series, Micropaleontology Press : American Museum of Natural History, 368 p.

BARBIN V. (1988) : La disparition de Chiasmolithus grandis (nannofossile calcaire) ne peut-être utilisée comme marqueur stratigraphique de la limite NP 17/NP 18 (Priabonien). C. R. Acad. Sci. Paris, t. 306, sér II, p. 297-300.

Berggren W.A, Kent D.V., Swisher C.C. III et Aubry M.-P. (1995) : A revised Cenozoic geochronology and chronostratigraphy. In Berggren W.A., Kent D.V., Aubry M.-P. et Hardenbol J. (eds) : Geochronology, time scales and global stratigraphic correlation. SEPM Special Publications, Tulsa, vol. 54, p. $129-212$.

Boulanger D. (1968) : Révision du Nummulitique de la Chalosse, du Béarn et du Bassin de l'Adour. Thèse Doct. d'Etat, Univ. Paris, 375 p.

GÉLY J.-P. et SzTRÀKos K. (2000) : L'évolution paléogéographique et géodynamique du Bassin aquitain au Paléogène : enregistrement et datation de la tectonique pyrénéenne. Géol. France, Ed. B.R.G.M. et S.G.F., Orléans, no 2, p. 31-57.

Kieken M. et Thibault C. (1975) : Note explicative et carte géologique de la France à $1 / 50000^{\mathrm{e}}$, Saint-Vincent-de-Tyrosse (n ${ }^{\circ}$ 975-976). B.R.G.M., Orléans, 46 p.

Lezaud L. (1967) : Contribution à l'étude des Nannofossiles calcaires de l'Eocène (Coccolithophoridés). Leur rôle stratigra- 
phique en Aquitaine sud-ouest (Chalosse, Béarn, Bas-Adour). Thèse Doct., Univ. Paris, 238 p., 25 pl.(inédite).

MARTINi E. (1971) : Standard Tertiary and Quaternary calcareous nannoplankton zonation. Proceedings 2d Planktonic Conference (Roma, 1970), Ed. Technoscienza, vol. 2, p. 739-785.

Martini E. et Müller C. (1986) : Current Tertiary and Quaternary calcareous nannoplankton stratigraphy and correlations. Newsl. Stratigr., Berlin, vol. 16, nº 2, p. 99-112.

Mathelin J.C. (1988) : Le Paléogène des falaises de Biarritz : révision biostratigraphique, paléoenvironnements et diapirisme. Mém. Sci. de la Terre, Univ. P. et M. Curie, Paris, $\mathbf{n}^{\circ}$ 88-23, $159 \mathrm{p}$.

Mathelin J.C. et SzTràkos K. (1993) : L'Eocène de Biarritz (Pyrénées Atlantiques, SW France). Stratigraphie et paléoenvironnement. Monographie des foraminifères. Cah. Micropal., Paris, n. sér., vol. $8, \mathrm{n}^{0} 1$, p. 5-85.

NoLF D. (1988) : Les otolithes de téléostéens éocènes d'Aquitaine (sud-ouest de la France) et leur intérêt stratigraphique. Mém. Acad. Roy. Belg., Cl. Sci., Bruxelles, $4^{\circ}, 2^{\mathrm{e}}$ sér., 19 (2), p. 1147.

Nolf D., Steurbaut E. et Cahuzac B. (ce volume) : Progrès récents dans la connaissance des gisements cénozoïques en Aquitaine méridionale (Chalosse, Béarn et Bas-Adour; SW France). Rev. Micropaléont., Paris.

OKADA H. et BUKRY D. (1981) : Supplementary modification and introduction of code numbers to the low-latitude coccolith biostratigraphic zonation (Bukry, 1973, 1975). Marine Micropal., Amsterdam, vol. 5, p. 321-325.

PERCH-Nielsen K. (1985) : Cenozoic calcareous nannofossils. In Bolli H.M., Saunders J.B. et Perch-Nielsen K. (eds) : Plankton Stratigraphy. Cambridge Earth Science Series, Cambridge University Press, vol. 1, 11, p. 427-554.

ShCherbiniva E.A. (2000) : Middle Eocene nannofossils and geological events of the northeastern peri-Tethys. In Schmitz B., Sundquist B. et Andreasson F.P. (eds) : Early Paleogene Warm Climates and Biosphere Dynamics. GFF, Stockholm, vol. 122 , part 1, p. 143-145.

SzTrÀKos K. (2000) : Les foraminifères de l'Eocène du Bassin de l'Adour (Aquitaine, France) : biostratigraphie et taxinomie. Rev. Micropaléont, Paris, vol. 43, $\mathrm{n}^{0}$ 1-2, p. 71-172.

Sztràkos K. et Castelltort S. (2001) : La sédimentologie et les foraminifères bartoniens et priaboniens des coupes d'Arguis (Prépyrénées aragonaises, Espagne). Incidence sur la corrélation des biozones à la limite Bartonien/Priabonien. Rev. Micropaléont., Paris, vol. 44, $\mathrm{n}^{\circ} 3$, p. 233-247.

Sztràkos K., GÉly J.P., Blondeau A. et Müller C. (1998) : L'Eocène du Bassin sud-aquitain : lithostratigraphie, biostratigraphie et analyse séquentielle. Géol. France, Ed. B.R.G.M et S.G.F., Orléans, n $^{0} 4$, p. 57-105.

Toumarkine M. et Luterbacher H.P. (1985) : Paleocene and Eocene planktic foraminifera. In Bolli H.M., Saunders J.B. et Perch-Nielsen K. (eds) : Plankton Stratigraphy. Cambridge Earth Science Series, Cambridge University Press, vol. 1, 5, p. 87-154.

Vandenberghe N., Brinkhuis H. et Steurbaut E. (2003) : The Eocene/Oligocene Boundary in the North Sea Area : A Sequence Stratigraphic Approach. In Prothero D.R., Ivany L.C. et Nesbitt E.A. (eds) : From Greenhouse to Icehouse : The Marine Eocene-Oligocene Transition, Columbia University Press, New York, chap. 24, p. 418-436.

van Morkhoven F.C.P.M., BERGgren W.A. et EDWARdS A.S. (1986) : Cenozoic cosmopolitan deep-water benthic Foraminifera. Bull. Centres Rech. Expl.-Prod. Elf-Aquitaine, Pau, mém. 11, $421 \mathrm{p}$.
VARoL O. (1992) : Sullivania a new genus of Palaeogene coccoliths. Journ. Micropal., London, vol. 11, no 2, p. 141-149.

\section{ANNEXE}

Liste alphabétique des nannofossiles calcaires trouvés dans la présente étude ou cités dans le texte : Blackites gladius (LOCKER, 1967) Varol, 1989 (Pl. 1, fig. 1 et 2 )

Bramletteius serraculoides GARTNER, 1969

Campylosphaera dela (Bramlette et Sullivan, 1961) Hay et Mohler, 1967

Chiasmolithus grandis (BRAMLETTE et RIEDEL, 1954) Radomski, 1968 (Pl. 1, fig. 25 et 26)

C. oamaruensis (DEFLANDRE, 1954) Hay, Mohler et Wade, 1966 (Pl. 1, fig. 17 et 18)

C. solitus (BRAMLETTE et SULLIVAN, 1961) Locker, 1968

Chiasmolithus sp. (PI. 1, fig. 8)

Clathrolithus spinosus MARTINI, 1961 (Pl. 1, fig. 23)

Clausicoccus subdistichus (ROTH et HAY in Hay et al., 1967) Prins, 1979

Coccolithus formosus (KAMPTNER, 1963) Wise, 1973 (Pl. 1, fig. 7)

Corannulus germanicus STRADNER, 1962 (Pl. 1, fig. 15)

Cribrocentrum reticulatum (GARTNER et SMITH, 1967) Perch-Nielsen, 1971 (Pl. 1, fig. 11)

Cyclicargolithus floridanus (ROTH et HAY in Hay et al., 1967) Bukry, 1971

C. neogammation (BRAMLETTE et WILCOXON, 1967) Bukry, 1971

Dictyococcites bisectus (HAY, MOHLER et WADE, 1966) Bukry et Percival, 1971 (Pl. 1, fig. 24)

Discoaster bifax BUKRY, 1971

D. saipanensis BRAMLETTE et RIEDEL, 1954 (Pl. 1, fig. 6)

D. wemmelensis ACHUTHAN et STRADNER, 1969

Hayella situliformis GARTNER, 1969

Helicosphaera bramlettei MÜLLER, 1970

H. compacta BRAMLETTE et WILCOXON, 1967 (Pl. 1, fig. 21)

H. papillata BUKRY et BRAMLETTE, 1969 (Pl. 1, fig. 5)

H. reticulata BRAMLETTE et WILCOXON, 1967

H. salebrosa PERCH-Nielsen, 1971 (Pl. 1, fig. 10)

H. wilcoxonii GARTNER, 1971 (Pl. 1, fig. 20) 
Isthmolithus recurvus DEFLANDRE, 1954

Lanternithus minutus STRADNER, 1962 (Pl. 1, fig. 13)

Lithostromation deflandrei LEZAUD, 1968 (Pl. 1, fig. 19)

Lophodolithus acutus BUKRY et PERCIVAL, 1971

Naninfula deflandrei PERCH-NIELSEN, 1968

Naninfula sp.

Neococcolithes minutus (PERCH-NIELSEN, 1967)

Perch-Nielsen, 1971 (Pl. 1, fig. 16)

Pedinocyclus larvalis (BUKRY et BRAMLETTE, 1969)

Loeblich et Tappan, 1973 (Pl. 1, fig. 12)

Pemma spp.

Pontosphaera prava (LOCKER, 1967) Romein, 1979

(Pl. 1, fig. 9)

P. aff. zigzag (ROTH et HAY, 1967) Aubry, 1986
Pontosphaera sp. (Pl. 1, fig. 4)

Reticulofenestra umbilica (LEVIN, 1965) Martini et Ritzkowski, 1968 (Pl. 1, fig. 3)

Sphenolithus furcatolithoides LOCKER, 1967

S. moriformis (BRÖNNIMANN et STRADNER, 1960)

Bramlette et Wilcoxon, 1967 (Pl. 1, fig. 14)

S. pseudoradians BRAMLETTE et WILCOXON, 1967

S. spiniger BUKRY, 1971 (PI. 1, fig. 27)

S. tribulosus RoTH, 1970

Sullivania gigas (BRAMLETTE et Sullivan, 1961) Varol, 1992

Sullivania sp. (PI. 1, fig. 22)

Zygrhablithus bijugatus (DEFLANDRE in DEFLANDRE et FERT, 1954) Deflandre, 1959 (PI. 1, fig. 28) 